\title{
Theoretical understanding of Galactic magnetic fields
}

\author{
Katia M. Ferrière \\ IRAP, Université de Toulouse, CNRS, 14 avenue Edouard Belin, F-31400 Toulouse, France
}

\begin{abstract}
I discuss our current theoretical understanding of the origin and evolution of interstellar magnetic fields in our Galaxy, with a focus on dynamo theory.
\end{abstract}

Keywords. ISM: magnetic fields

It is likely that the very first cosmic magnetic fields were generated before the formation of galaxies - for instance, through a battery effect. These pre-galactic magnetic fields would then have been amplified in galaxies by several mechanisms, starting with (1) the compression of field lines during the collapse of protogalaxies, and continuing with (2) the stretching of field lines by the large-scale differential rotation of galaxies and (3) the production of magnetic field perpendicular to the prevailing field by small-scale helical turbulence (a process known as the alpha effect). Together the last two mechanisms can amplify magnetic fields at a very fast (initially exponential) rate: this is the essence of dynamo action in galaxies.

Mean-field dynamo theory provides an evolution equation for the mean (or large-scale) magnetic field, $\langle\vec{B}\rangle$ - the dynamo equation - wherein the effects of turbulent motions are parameterized by means of two tensors: $\alpha$, which mainly describes the alpha effect, and $\beta$, which mainly describes turbulent magnetic diffusion.

When the large-scale velocity and the $\alpha, \beta$ tensors are prescribed, the dynamo equation is linear in $\langle\overrightarrow{\boldsymbol{B}}\rangle$, which, therefore, grows (or decays) exponentially with time. In addition, $\langle\overrightarrow{\boldsymbol{B}}\rangle$ generally has the following properties: (1) In galactic disks, the azimuthal component of $\langle\overrightarrow{\boldsymbol{B}}\rangle$ dominates, while its vertical component is very small. (2) In an axisymmetric galaxy, axisymmetric $(m=0)$ modes are easiest to amplify; in the presence of an external disturbance, bisymmetric $(m=1)$ modes can possibly be amplified, while in the presence of a spiral or bar, quadrisymmetric $(m=2)$ modes can possibly be amplified. (3) Under typical galactic conditions, both symmetric and antisymmetric (with respect to the midplane) modes are amplified; if the disk dominates, symmetric modes tend to grow faster, while if the halo dominates, antisymmetric modes tend to grow faster.

When the magnetic field has grown strong enough, it starts having a back-reaction on the turbulence, which "quenches" $\alpha$ and causes the field growth to saturate. The conventional approach is to consider that back-reaction starts when the mean field approaches equipartition with the turbulence. However, several authors have pointed out that the small-scale field grows much faster than the mean field, such that back-reaction actually starts when the small-scale field approaches equipartition, i.e., when the mean field is still a huge factor ( $\sim \sqrt{\mathrm{Rm}}$, with $\mathrm{Rm}$ the magnetic Reynolds number) below equipartition (catastrophic $\alpha$-quenching). In reality, the mean field can keep growing after the small-scale field has reached equipartition, with the temporal evolution of $\alpha$ governed by the conservation of magnetic helicity (dynamical $\alpha$-quenching).

The galactic dynamo problem has also been tackled through direct numerical simulations, the most recent of which cover a full galaxy (disk + halo). 